\title{
The Athena Semi-Automated Karyotyping System ${ }^{1}$
}

\author{
Lucas J. van Vliet, ${ }^{2}$ Ian T. Young, and Brian H. Mayall \\ Pattern Recognition Group of the Faculty of Applied Physics, Delft University of Technology, 2628 CJ Delft, \\ The Netherlands (L.J.v.V., I.T.Y.); Laboratory for Cell Analysis, University of California, San Francisco, \\ California 94143 (B.H.M.) \\ Received for publication June 7, 1989; accepted September 1, 1989
}

\begin{abstract}
In this article we describe Athena, a system that provides for semi-automated karyotyping of metaphase spreads. The system is based upon the Macintosh II computer. It uses software that is written entirely in $C$ and consists of approximately 200 Kbytes of executable code. Athena provides automated segmentation of metaphase images into individual chromosomes, automated measurements on each banded chromosome, and automated classification into the standard Paris-convention karyotype. Furthermore, the system provides the ability to construct one or more chromosome
\end{abstract}

data bases to represent the types of metaphase spreads and staining techniques that may be used in a given laboratory. Because we believe that it is impossible to construct a system that can achieve perfect segmentation, perfect separation of touching and overlapping chromosomes, perfect localization of the centromeres, and perfect classification, the system offers the possibility for interaction at each of the above stages using the well-accepted Macintosh user interface.

Key terms: Automated chromosome analysis, karotyping, pattern recognition
For more than 25 years medical scientists, physical scientists, mathematicians, engineers, and computer scientists have attempted to automate the analysis of metaphase chromosome spreads. In an important sense automated karyotyping was one of the first problems in digital image analysis and pictorial pattern recognition. That there are still a number of on-going and healthy research efforts in this field-approximately 25 years after the publication of Ledley $(16,17)$ and Neurath (28)-shows that the problem is both exquisitely difficult and extremely important. It should be remembered, however, that it was less than 35 years ago that the correct number of chromosomes in the normal human chromosome complement, 46, was first enumerated (36). Furthermore, accurate and reproducible identification of each of the 24 possible chromosome classes became possible only after development of banding stains by Caspersson and his co-workers $(4,5)$ in 1970 .

Technical developments of the past 10 years have contributed significantly to automated karyotyping, and there now are more than ten companies producing systems for computer processing of digitized chromosome images. These systems range from those that can only be described as "electronic scissors" to systems that combine metaphase finding as well as pattern classification of banded chromosomes to achieve a result that requires a minimum of human interaction.
We (Young, Mayall, and the Delft group) have been involved for more than 15 years in research into various aspects of the quantitative and automated analysis of digitized chromosome images. This research has covered virtually all aspects of the quantitative analysis of chromosome images and includes the following:

1. Metaphase finding (3)

2. Automatic focusing $(15,25)$

3. Analysis of banding patterns $(11,34,35,38)$

4. Centromere location $(6,27,38)$

5. Chromosome aberrations $(20,21,22,43)$

6. Accurate DNA measurements from digital images $(14,23,24,26,27)$

7. DNA species within chromosomes (41)

\footnotetext{
${ }^{1}$ This work was partially supported by the University of California Program for Analytical Cytology, AMOCO Technology Company (Naperville, Illinois), and the Commission of the European Communities through the Medical and Health Research Program, project number II.1.1/13, and was performed under the auspices of the U.S. Department of Energy by the Lawrence Livermore National Laboratory under contract number W-7405-ENG-48.

${ }^{2}$ Address reprint requests to Lucas J. van Vliet, Pattern Recognition Group, Faculty of Applied Physics, Delft University of Technology, Lorentzweg 1, 2628 CJ Delft, The Netherlands.
} 
Based on these developments, we decided in 1987 that we were in a position to construct a karyotyping workstation based upon a modern, low-cost computer "platform," the Apple Macintosh II personal computer. With the exception of automated metaphase finding, we had assembled the "software" components required to generate a karyogram automatically, given a metaphase cell. The components consisted of the following:

1. Image segmentation to find individual objects;

2. Procedures for eliminating non-chromosome-like objects;

3. Procedures to separate touching chromosomes;

4. An accurate method for chromosome rotation;

5. An accurate procedure to locate centromeres;

6. An intuitive but quantitative way to describe banding patterns;

7. A context-sensitive classification procedure based upon relative length, centromeric index, and band description.

\section{MATERIALS AND METHODS}

The Athena workstation consists of a Macintosh II computer (Apple Computer, Cupertino, CA) with 16 $\mathrm{MHz}$ MC68020 processor, MC68881 floating point coprocessor (the Macintosh Ilcx provides a MC68030 processor, a MC68882 floating point co-processor, and a $20 \%$ improvement in speed), 5 MBytes RAM, 80 MBytes high-speed hard disk, Cohu 4810 CCD camera (Cohu, San Diego, CA), small black and white video monitor, QuickCapture (Data Translation, Marlboro, MA) frame grabber, and a Spectrum/8 Series II (SuperMac Technology, Sunnyvale, CA) integrated 8-bit deep color and grey-level video card driving a SuperMac 19 inch high-resolution color monitor displaying $1,024 \times$ 768 pixels. A hard copy can be produced either by PostScript-coded halftone printing on a LaserWriter or true grey-level printing using a dry-silver paper laser printer. The software development environment for this project is MPW (1) offering C, Pascal, object-oriented Pascal, and assembly language.

Athena is organized around the concept of a case study. A case study represents the analysis of the cytogenetic material from a single individual. The user initiates a session with the program by "double-clicking" (or opening) the program icon shown in Figure 1. Thus, the user initiates and interacts with the program along lines defined in the Macintosh user protocol (2).

Details of Athena operation are described in the companion article by Mayall et al. (37). We now describe technical details of the three major steps in karyotyping:

1. Segmentation of the metaphase spread image;

2. Analysis of individual chromosome images;

3. Classification of the chromosomes into a karyogram.

\section{Segmentation}

The fundamental technique used for segmentation is a combination of shading correction, thresholding, and

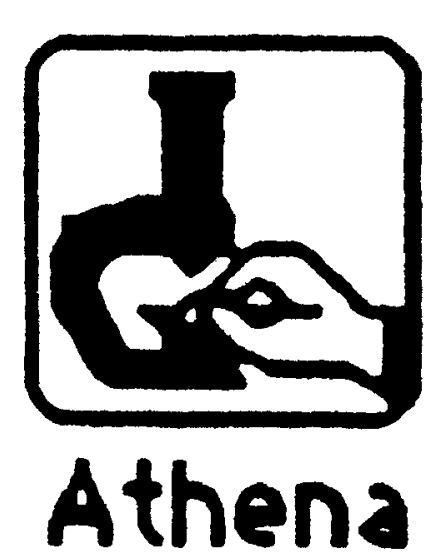

Flg. 1. The Icon for the Athena program.

binary image operations. Shading correction is offered as an option to remove (possible) effects associated with non-uniform camera sensitivity or illumination conditions, and to diminish the effects of non-specific background staining. Shading correction is accomplished through the use of the grey-level morphologic filtering operations dilation (maximum) and erosion (minimum). The estimate of the background shading $\mathbf{S}$ from an original image $\mathbf{I}$ is given by

$$
\mathbf{S}=\operatorname{Min}_{\mathbf{n}}\left(\operatorname{Max}_{\mathbf{n}}(\mathbf{I})\right)
$$

where $\mathbf{n}$ is the neighborhood (support) of the filter. In this case the filter is always square of size $\mathbf{n}$, and the value of $\mathbf{n}$ should be chosen to be larger than the diameter of the largest object (usually an interphase nucleus) to be found in the image. The corrected image is then given by

$$
\mathbf{C}=\text { WHITE }+(\mathbf{I}-\mathbf{S})
$$

where WHITE is simply the maximum possible value (usually 255) in the absorption image. A threshold is used to convert the image into a binary representation where the chromosomes and other objects are black (1) and the background is white $(0)$. Various possible algorithms to select the threshold are displayed in the Segmentation window (Fig. 2). The threshold selection algorithms have been described previously $(33,40,42)$.

Binary image filtering is used to reduce the inevitable "false-positives" and "false-negatives" produced by thresholding. The operations erosion, dilation, propagation, exclusive-or, skeletonizing, and anding are used to eliminate small artifacts and large objects, to fill holes inside chromosomes, and to separate touching chromosomes. The actual algorithms used to implement these operations take advantage of techniques that we have developed (39).

Chromosomes that touch only slightly are separated by eroding the binary image a few times and then computing the background skeleton (exo-skeleton). This exo-skeleton forms dividing lines that separate the touching chromosomes. This technique is illustrated in 


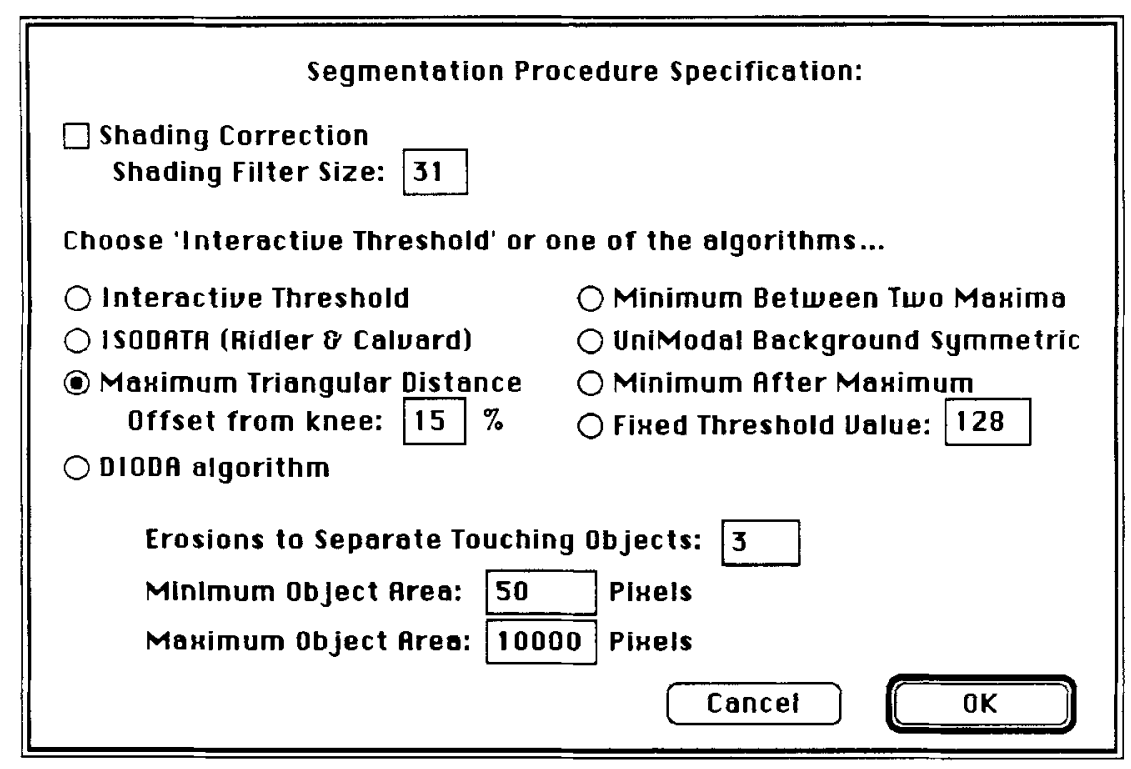

FIG. 2. The user chooses the parameters associated with shading correction, thresholding, and binary image filtering.

Figure 3. At the completion of the segmentation phase the user can correct the remaining errors through the use of the mouse and a menu interface (42).

\section{Analysis}

The analysis phase consists of 1 ) determining the orientation of the major axis of each chromosome and rotating the individual chromosomes so that their major axes are vertical (13), 2) determining the centromere position using the technique developed by Visser (38), and 3) measuring the chromosome length, the centromeric index, and the band descriptions as described by Visser (38).

Rotation of chromosomes. The rotation angle is estimated from the first- and second-order moments of inertia of the binary image associated with the chromosome. The classic technique of bilinear interpolation is used to perform the rotation of the individual greylevel chromosome images given the rotation angle. Care is taken to implement the rotation in such a way that the DNA distribution is not distorted (12).

Centromere detection and centromeric index. Automatic centromere location has proven to be a difficult task, and no available technique achieves complete success. Many techniques search for a pair of concavities along the chromosome contour. Pairs of opposite concavities then form candidates for the centromere position. Athena's automatic centromere detector searches for two points having the shortest distance between the left and the right contour of a chromosome (see Fig. 4). In order to avoid detection at the ends of the chromosomes (telomeres), a certain distance from the top and bottom of the chromosomes is skipped. The user specifies this value as a percentage.
The default value of $15 \%$ approaches the centromeric index of the acrocentric chromosomes of classes 13 through 15. All techniques using the morphologic characteristic of a centromere-indentation at two sides along the medial axis - will degrade in performance for images with many clusters of touching and overlapping chromosomes that have to be separated manually, as the "artificial" boundary does not reflect the "natural" boundary.

We define the centromeric index as

$$
\mathrm{CI}=\frac{\text { Length of the short arm }(\mathrm{P} \text { terminal })}{\text { Total length of chromosome }} \times 1000
$$

This produces a number in the interval $0<\mathbf{C I}<500$.

Measuring the band parameters. Assuming absorption imagery, bands are considered as dark regions of the chromosome. An optical density-based threshold selects the dark parts as regions potentially bearing a band. In this way the detection of vague bands and vague connections between clearly separated bands is avoided. The default value of the threshold is set to 0.1 , which corresponds to a transmittance of $=80 \%$ in the band relative to the background.

The selected regions are then Laplace filtered along the main (medial) axis. This second-order derivative filtering leads to the detection of hills (negative areas) and valleys (positive areas) in the grey value image. All hill-points are labeled, and every set of connected points forms a candidate for a band. For each band, parameters are calculated such as area, total optical density, begin, end, and middle position relative to the top. From this information a subset of the bands is extracted and used for classification. Athena uses the 

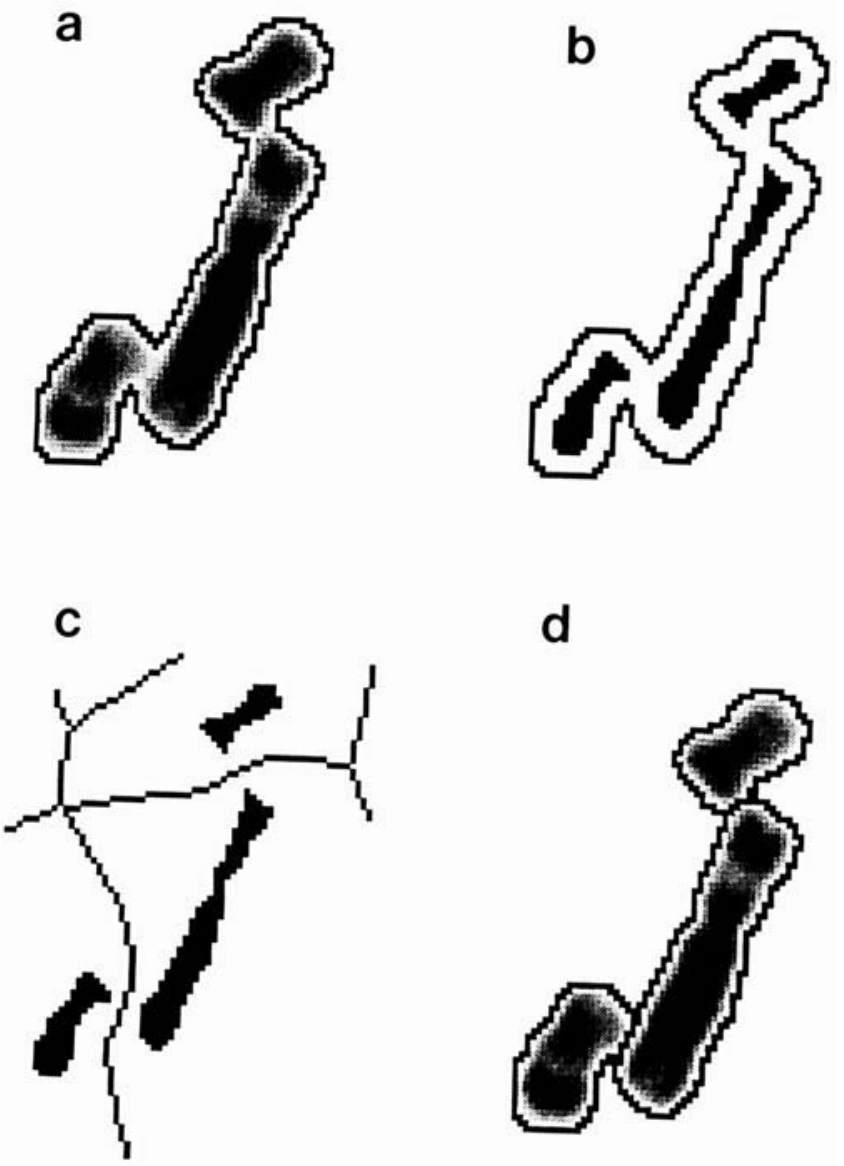

FIG. 3. Separation of touching chromosomes. a: Cluster of three touching chromosomes and circumscribing edges. b: Original edges and binary mask of the cluster after a couple of erosions-the cluster is split into three bodies. c: Exo-skeleton of the eroded mask. d: The exo-skeleton is used to decompose the cluster into three individual chromosomes.

central position of the following bands as the chromosome band features:

1. Darkest band on the chromosome

2. Band having the largest area

3. Distal band on the short arm

4. Distal band on the long arm

5. Band closest to the centromere on the long arm.

After rotation, centromere localization, and band measurement, the seven features-length, CI, and the five band positions-are assembled (see Fig. 5). The user may examine the data by summoning the Features window from the Windows menu.

\section{Classification}

The final phase of processing is the classification of the chromosomes on the basis of the measured features. Athena allows the user to indicate the name of the database that will serve as the standard for classification (the training set), the name of the database to
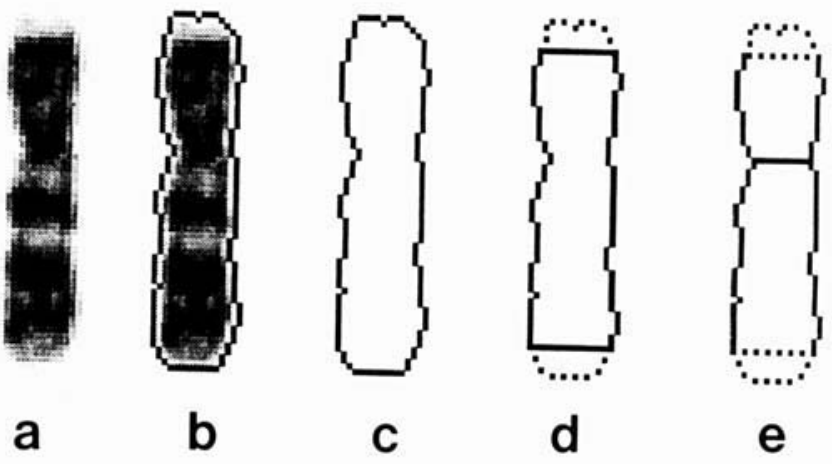

FIG. 4. Centromere finding using the shortest distance method (38). a: Grey-level chromosome image (chromosome 7). b: Grey-level chromosome image and corresponding edges. c: Chromosome edge image. d: Skip 15\% from the telomeres. e: Find the shortest distance between the opposite contours, i.e., centromere position.

which results may be added based on the analysis of new metaphases, and some parameters associated with the classification algorithms.

Before classification we have measured the feature vector, $\mathbf{x}$, for each unclassified chromosome. Further, we know the estimated frequency distribution of a feature vector for each of the 24 chromosome classes $\mathbf{p}\left(\mathbf{x} \mid \omega_{\mathbf{j}}\right)$ (from the training database) and the a priori probability for a chromosome to belong to a class $j$, $\mathbf{P}\left(\omega_{\mathrm{j}}\right)$. The a priori probability is based upon biological information. (A healthy human being has 22 homologous autosomal pairs and 2 sex chromosomes.) This information offers the opportunity to use the non-parametric Bayes' rule to calculate the a posteriori probability $\mathbf{P}\left(\omega_{\mathbf{j}} \mid \mathbf{x}\right)$ that each individual chromosome belongs to class $\omega_{j}$ (see 8):

$$
\begin{aligned}
& P\left(\omega_{j} \mid x\right)=\frac{p\left(x \mid \omega_{j}\right) P\left(\omega_{j}\right)}{p(x)} \\
& p(x)=\sum_{j=1}^{24} p\left(x \mid \omega_{j}\right) P\left(\omega_{j}\right)
\end{aligned}
$$

This rule can be shown to produce, on average, the classification with the minimum numbers of errors.

For practical reasons we assume that the features are independent, so equation $4 a$ can be rewritten as

$$
\mathbf{P}\left(\omega_{j} \mathbf{x}\right)=\frac{\prod_{n=1}^{7} \alpha_{n} p\left(\mathbf{x}_{n} \mid \omega_{j}\right) \mathbf{P}\left(\omega_{j}\right)}{\mathbf{P}(\mathbf{x})}
$$

where $x_{n}$ is an element of the feature vector $\mathbf{x}$, that is, one of the seven measurements, and $\left\{\alpha_{n} \mid n=1, \ldots, 7\right\}$ are weighting coefficients. The additional coefficients $\left\{\alpha_{n}\right\}$ are not part of the Bayes' test but are available to the user to reflect local peculiarities of use. The Bayes' test is used by setting $\alpha_{n}=1$ for $n=1,2, \ldots, 7$. The setting of the coefficients are selected through a standard Macintosh dialog box.

Estimating the frequency distributions. The frequency distributions (or class conditional probability density functions) are estimated from a learning set of 


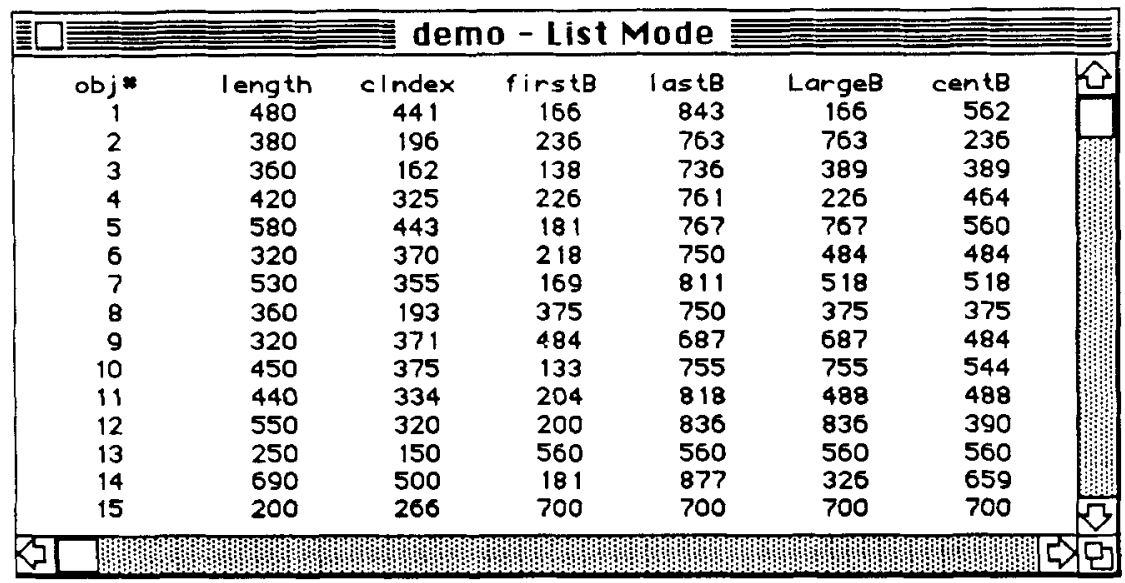

FIG. 5. The measurements per chromosome are displayed and stored in a list mode. Object number refers to the order in which the chromosomes were found in the image and not to their class. The positions of the bands are relative to a normalized length of 1,000 per chromosome. Thus object 1 has a last band at $84.3 \%$ of the distance from the top of the chromosome. Six of the seven measurements are shown here. The last measurement is seen by "scrolling" to the right. patterns (database) with known class membership. Because the band features used are non-parametric, the problem of estimating $\mathbf{p}\left(\mathbf{x} \mid \omega_{\mathrm{j}}\right)$ cannot be reduced to the problem of estimating the parameters of a distribution such as the mean and standard deviation of a hypothesized Gaussian distribution.

Athena uses the histogram approach to transform a data set into a probability density function. The range of each feature $\mathbf{x}_{\mathbf{i}}$ of vector $\mathbf{x}$ is divided into a fixed number of equal intervals or bins. The number of data points falling into each bin is counted and forms the basis for the probability estimate. The number of bins strongly depends on the size of the learning set and the underlying distribution. For Gaussian distributions the number of bins should be about $\sqrt{N}$ (where $N$ is the size of the learning set). Because the distribution of each chromosome's length is approximately Gaussian and fills about one-third of the total range, $3 \sqrt{N}$ is a good estimate for the total number of bins to use. As an example, if we have 40 chromosomes per class, then the number of bins should be about $3 \sqrt{40} \approx 20$.

Context-sensitive classification. After computing the a posteriori probabilities for each chromosome, a context-sensitive classifier assigns the chromosomes to the possible classes. It is assumed that the metaphase contains two copies of each autosome and two sex chromosomes, XX for females and XY for males. An exception is made when there is an abnormal number of chromosomes in the cell, e.g., Down's syndrome. The classification is a two-step process.

In the first step, all chromosomes are ordered in decreasing length, building a list with the longest chromosomes first. The chromosome classes are also ordered in a list of decreasing length: 1 through $7, \mathrm{X}, 8$ through 22, and $\mathrm{Y}$. These two lists are then associated with each other: the first and second chromosomes from the length list with chromosomes class 1 , the next two chromosomes with chromosome class 2 , and so forth.

After this initial classification based upon length, we assign cost factors to the surrounding classes that make a transfer to another class over longer distances less likely. Distance is defined as the absolute difference in class number between start class and end class. Thus, moving a chromosome from class 2 to class 6 is a distance of 4 . Athena (in the current version) assigns no cost $(\mathrm{C}=0)$ to classes with a distance smaller than or equal to a pre-defined window size (default size $=3$ ) from the current class leaving the a posteriori probabilities $P\left(\omega_{\mathbf{j}} \mid \mathbf{x}\right)$ for $\mathbf{j}$ in (class-window) $\leq \mathbf{j} \leq$ (class + window) unchanged. An infinite cost $(\mathrm{C}=x)$ is assigned to all classes with a distance larger than the window size from the current class making the a posteriori probabilities $\mathbf{P}\left(\omega_{j} \mid \mathbf{x}\right)$ zero for all $\mathbf{j}$ in the intervals: $\mathbf{0} \leq \mathbf{j}<$ (class-window) and (class + window) $<$ $\mathbf{j} \leq \mathbf{2 4}$ with (class-window) $\geq \mathbf{0}$ and (class + window) $\leq 24$.

All chromosomes are initially attached to the class with the highest a posteriori probability, still available, according to the Bayes' rule. The limit of two chromosomes per class is taken into account resulting in transfers where more than two chromosomes per class are found. This procedure continues until all chromosomes are either classified or rejected. A chromosome will be rejected if its a posteriori probability for the remaining classes is lower than a given minimum probability threshold (default $P_{\min }=.005$ ).

The default values were chosen for standard metaphase spreads as obtained through amniocentesis and $\mathrm{G}$ band staining. Of particular importance is the ability to construct a database as well as a classifier that is "tuned" to the procedures and material within a given laboratory environment. That is, Athena can be used to 
Table 1

Accuracy of the Five Centromere Location Schemes Described in the Text ${ }^{\text {a }}$

\begin{tabular}{|c|c|c|c|c|c|}
\hline $\begin{array}{l}\text { Chromosome } \\
\text { No. }\end{array}$ & $\begin{array}{c}\text { Shortest } \\
\text { distance, \% }\end{array}$ & $\begin{array}{l}\text { Convex } \\
\text { hull, } \%\end{array}$ & $\begin{array}{c}\text { Width } \\
\text { profile, } \%(1)\end{array}$ & $\begin{array}{c}\text { Width } \\
\text { profile, } \%(2)\end{array}$ & $\begin{array}{c}\text { Convex } \\
\text { profile, } \%\end{array}$ \\
\hline 1 & 76.5 & 52.9 & 73.5 & 64.7 & 85.3 \\
\hline 2 & 80.0 & 71.4 & 77.1 & 62.9 & 91.4 \\
\hline 3 & 92.3 & 43.6 & 74.4 & 59.0 & 78.9 \\
\hline 4 & 100.0 & 72.1 & 83.7 & 69.8 & 90.5 \\
\hline 5 & 95.6 & 73.3 & 95.6 & 44.4 & 91.1 \\
\hline 6 & 89.2 & 75.5 & 83.8 & 86.5 & 91.9 \\
\hline 7 & 87.8 & 82.9 & 90.2 & 87.8 & 87.8 \\
\hline 8 & 92.3 & 97.4 & 97.4 & 76.9 & 97.4 \\
\hline 9 & 81.1 & 78.4 & 94.6 & 81.1 & 89.2 \\
\hline 10 & 89.7 & 92.3 & 82.1 & 74.4 & 87.2 \\
\hline 11 & 78.6 & 73.2 & 85.7 & 61.9 & 76.2 \\
\hline 12 & 83.3 & 69.0 & 83.3 & 73.8 & 83.3 \\
\hline 13 & 95.3 & 92.1 & 41.9 & 83.7 & 66.7 \\
\hline 14 & 97.5 & 72.5 & 17.5 & 85.0 & 52.6 \\
\hline 15 & 97.3 & 92.1 & 34.2 & 92.1 & 72.7 \\
\hline 16 & 83.3 & 88.4 & 86.0 & 76.7 & 100.0 \\
\hline 17 & 82.1 & 71.8 & 35.9 & 84.6 & 82.1 \\
\hline 18 & 92.5 & 92.7 & 36.6 & 92.7 & 95.1 \\
\hline 19 & 53.5 & 82.9 & 48.8 & 67.4 & 83.3 \\
\hline 20 & 69.2 & 86.5 & 74.4 & 69.2 & 94.9 \\
\hline 21 & 86.8 & 39.4 & 34.2 & 71.1 & 50.0 \\
\hline 22 & 62.5 & 63.9 & 57.5 & 35.0 & 61.8 \\
\hline $\mathrm{X}$ & 96.6 & 82.2 & 100.0 & 89.7 & 96.6 \\
\hline $\mathrm{Y}$ & 80.0 & 80.0 & 40.0 & 80.0 & 60.0 \\
\hline Average & 85.1 & 76.1 & 67.9 & 73.8 & 81.9 \\
\hline
\end{tabular}

anine hundred twenty-four chromosomes (32) were used in this study. A deviation of more than one pixel from the position indicated by the "expert" was considered as an error in centromere location.

construct de novo a database specific for a particular application or laboratory.

\section{RESULTS}

In this section we present some of the results achieved with the techniques incorporated in Athena. Some of these results-such as the centromere detection accuracy and the classification accuracy-were generated in previous studies. The procedures, however, have been incorporated unchanged into this package and thus are representative of the results that can be achieved.

\section{Centromere Location}

In this comparison we look at five techniques for determining the position of the centromere of banded chromosomes. The first technique is based upon the smallest distance across the chromosome (38). The second technique uses the maximum deviation from the convex hull of the chromosome to the chromosome contour itself (31). The third technique uses a polynomial fit to the width profile of the chromosome to find the centromere (44). The profile is filtered and the deepest minimum is used as the centromere; if no clear minimum exists-as, for example, in many acrocentric chromosomes-the inflection point is used to determine the centromeric position. The fourth technique uses the difference between a "standard" width profile and the measured profile (18). The last method, the convex profile technique, is based upon the deviation between the width profile and its convex hull (30).

In all of the comparisons, the chromosomes were first rotated and straightened (13). The details of the entire experiment are described in de Muinck Keizer (7). The results are shown in Table 1 . In a prior study (7), an additional technique based upon the measurement of local curvature (9) was examined. This technique led to an average accuracy of $15 \%$ and was thus discarded. The shortest distance method thus provides a significant improvement in the correct identification of the centromere position when compared to the other techniques. Its accuracy, however, remains below $90 \%$. This indicates that, on the average, at least five chromosomes per metaphase would have to be corrected interactively.

\section{Classification Accuracy}

The complete procedure for classification described above has been tested on the same set of 924 chromosomes used for the centromere study. The test set was identical with the learning set because of the relatively small number of chromosomes per class $(\approx 20)$. The results, starting from the correct centromere positions, are shown in Table 2 together with a comparison to Granum's WDD functions (10) on the same chromosomes.

Further, the two techniques-Laplace/band description versus WDD functions-were also compared on a 
Table 2

Results of the Classification of 924 Chromosomes $^{\text {a }}$

\begin{tabular}{lcc}
$\begin{array}{l}\text { Leiden } \\
\text { data set }\end{array}$ & $\begin{array}{c}\text { Laplace/band } \\
\text { descriptors, } \%\end{array}$ & $\begin{array}{c}\text { WDD } \\
\text { functions, } \%\end{array}$ \\
\hline Error rate & 4.0 & 4.1 \\
Rejects & 0.0 & 1.6 \\
\hline
\end{tabular}

an this experiment the test set equaled the learning set $(34,35)$ for both classification techniques.

much larger data set obtained from Lundsteen (19). The results are given in Table 3 .

In the Laplace/Band Descriptors technique, 7,284 chromosomes were classified, and the test set did not equal the learning set (34). Bent chromosomes were classified but not straightened. In the WDD technique, 6,985 chromosomes were classified, and the test set did equal the learning set. Furthermore, bent chromosomes were excluded from the classification procedure.

The use of the learning set as the test set in the testing of the WDD function approach and the exclusion of bent chromosomes means that the value of $2.1 \%$ must be considered as highly optimistic. It is not possible for us to conclude that the technique we have implemented-a context-sensitive classifier based upon bands identified by a form of Laplace filtering - is better than the WDD classifier. We can conclude, however, that it offers a reasonable accuracy. Further, the description developed by this classifier is much closer to the verbal description offered by cytogeneticists and embodied in the Paris convention (29). It is possible to read the numbers in Figure 5 and know-for any given chromosome-what that will mean in terms of banding pattern.

\section{DISCUSSION}

We have described in this article a software system for the (semi-)automatic analysis of metaphase spreads based upon a Macintosh II personal computer. This system takes full advantage of the hardware facilities in the computer: the 32-bit address space, the high-speed disk, the floating point co-processor (required for chromosome rotation and straightening), the 8-bit deep display for color and grey levels, and the mouse-based user interface. For an indication of the total performance the reader is referred to the companion article by Mayall et al. (37). In testing Athena on a variety of metaphase spreads, the actual processing time for automatic segmentation and classification averaged only 90 seconds on a Macintosh II and would be even less on a Macintosh IIcx.

\section{ACKNOWLEDGMEN'TS}

We wish to acknowledge the help and advice of our colleagues in Leiden, Copenhagen, Edinburgh, and Livermore.

\section{LITERATURE CITED}

1. APDA--Apple Programmer's Development Association: Macintosh Programmer's Workshop Reference-Version 2.0, Renton, Washington, 1988.
Table 3

Results of the Classification Experiment on the Lundsteen et al. (19) Data Set

\begin{tabular}{lcc}
$\begin{array}{l}\text { Copenhagen } \\
\text { data set }\end{array}$ & $\begin{array}{c}\text { Laplace/band } \\
\text { descriptors, } \%\end{array}$ & $\begin{array}{c}\text { WDD } \\
\text { functions, } \%\end{array}$ \\
\hline Error rate & 11.5 & 2.1 \\
Rejects & 0.0 & 0.1 \\
\hline
\end{tabular}

2. Apple: Inside Macintosh Vol. I-III,IV,V, Addison-Wesley Publishing Company Inc., Reading, MA, 1985-1987.

3. Bishop RP, Young IT: The automated classification of mitotic phase for human chromosome spreads. J Histochem Cytochem 25(7):730-740.

4. Caspersson T, Zech L, Johansson C: Differential banding of alkylating fluorochromes in human chromosomes. Exp Cell Res 60: $315-319,1970 \mathrm{a}$.

5. Caspersson T, Zech L, Johansson C, Modest EJ: Identification of human chromosomes by DNA-binding fluorescent agents. Chromosoma $30: 215,1970 \mathrm{~b}$.

6. de Muinck Keizer M, Groen FCA, Young IT, Smeulders AWM: An objective comparison of various centromere determination techniques: Proceedings of the Vth European Chromosome Analysis Workshop, Heidelberg, West Germany, 1983.

7. de Muinck Keizer M: An objective comparison of various centromere determination techniques. Master's thesis, Department of Applied Physics, Delft University of Technology, (in Dutch), (Thesis supervisors: F.C.A. Groen and I.T. Young), 1984.

8. Duda RO, Hart PE: Pattern Classification and Scene Analysis. John Wiley-Interscience, New York, 1973.

9. Gallus G, Neurath PW: Improved computer chromosome analysis incorporating preprocessing and boundary analysis. Physics Med Biol 15:435-445, 1970

10. Granum E, Gerdes T, Lundsteen C: Simple weighted density distributions, WDD's for discrimination between G-banded chromosomes. Proceedings of the IVth European Chromosome Analysis Workshop, Edinburgh, 1981.

11. Granlund GH, Zack GW, Young IT, Eden M: A technique for multiple-cell chromosome karyotyping. J Histochem Cytochem 24(1):160-167, 1976.

12. Groen FCA: Ph.D. thesis, Analysis of DNA based measurement methods applied to human chromosome classification, Faculty of Applied Physics, Delft University of Technology, The Netherlands, 1977.

13. Groen FCA, Verbeek PW, Zee GA van der, Oosterlinck A: Some aspects concerning computation of chromosome banding profiles. Proceedings of the IIIrd International Joint Conference on Pattern Recognition, Coronado, California, pp 547-550, 1976.

14. Groen FCA, van der Ploeg M: DNA cytophotometry of human chromosomes. J Histochem Cytochem 27:435-440, 1979.

15. Groen FCA, Young IT, Ligthart G: A comparison of different focus funetions for use in autofocus algorithms. Cytometry 6:81-91, 1985.

16. Ledley RS: High speed automatic analysis of biomedical pictures. Science 146:216-223, 1964.

17. Ledley RS, Rotolo LS, Golab TJ, Jacobsen JD, Ginsberg MD, Wilson JB: FIDAC-Film input to digital automatic computer and associated syntax directed pattern recognition programming system. In: Optical and Electro-Optical Information Processing, Tippet JI, Beckowitz D, Clapp L, Koester C, Vanderburgh A (eds). MIT Press, Cambridge, MA, 1965, pp 591-631.

18. Lucas JN, Gray JW, Peters DC, Van Dilla MA: Centromeric index measurement by slit-scan flow cytometry. Cytometry 4(2):109116, 1983

19. Lundsteen C, Philip J, Granum E: Quantitative analysis of 6985 digitized trypsin G-banded himian metaphase chromosomes. Clin Genet 18:335-370, 1980.

20. Mayall $\mathrm{BH}$, Carrano AV, Rowley JD: DNA cytophotometry of chromosomes in a case of chronic myelogenous leukemia. Cancer Res 37:3590-3593, 1974. 
21. Mayall BH, Carrano AV, Golbus MS, Conte FA, Epstein CJ: DNA cytophotometry in prenatal cytogenetic diagnosis. Clin Genet 1: 273-276, 1977a.

22. Mayall BH, Carrano AV, Moore DH, Rowley JD: Quantification by DNA-based cytophotometry of the $9 q+122 q$-chromosomal translocation associated with chronic myelogenous leukemia. Cancer Res 37:3590-3593, 1977b.

23. Mayall BH, Carrano AV, Moore DH, Ashworth LK, Bennett DE, Mendelsohn ML: The DNA-based human karyotype. Cytometry 5:376-385, 1984 .

24. Mendelsohn ML, Hungerford DA, Mayall BH, Perry BH, Conway TJ, Prewitt JMS: Computer-oriented analysis of human chromosomes. II. Integrated optical density as a single parameter for karyotype analysis. Ann NY Acad Sci 157:376-393, 1969.

25. Mendelsohn ML, Mayall BH: Computer-oriented analysis of human chromosomes. III. Focus. Computers Biol Med 2:137-150, 1972.

26. Mendelsohn ML, Mayall BH, Bogart E, Moore DH, Perry BH: DNA content and DNA-based centromeric index of the 24 chromosomes. Science 179:1126-1129, 1973

27. Mendelsohn ML, Bennett DE, Bogart E, Mayall BH: Computeroriented analysis of human chromosomes. IV. Deoxyribonucleic acid based centromeric index. I Histochem Cytochem 22:554$560,1974$.

28. Neurath PW, et al.: Human chromosome analysis by computeran optical pattern recognition problem. Ann NY Acad Sci 128: 1013-1028, 1965.

29. Paris Conference: Standardization in human cytogenetics. Birth Defects VIII:7.

30. Piper J, Granum E, Rutovitz D, Ruttledge H: Automation of chromosome processing. Signal Processing 2:203-221, 1980.

31. Piper J: Finding chromosome centromeres using boundary and density information. In: Digital Image Processing, Simon J-C, Haralick RM (eds). D. Reidel, Dordrecht, The Netherlands, 1981, pp 511-518.

32. Ploeg $M$ van der, Duyn $\mathrm{P}$ van, Ploem JS: High resolution scanning densitometry of photographic negatives of human metaphase chromosomes, Parts I and II. J Histochem Cytochem 42 : 9-46, 1974.

33. Ridler TW, Calvard S: Picture thresholding using an interactive selection method. IEEE Trans Systems, Man Cybernetics SMC. $8(8): 630-632,1978$.
34. Ten Kate TK, Groen FCA, Young IT, van der Ploeg M, Pearson PL, Gerdes T, Lundsteen C: The Delft classification technique on some difficult chromosome classes. Proceedings of the Vth European Chromosome Analysis Workshop, Heidelberg, West Germany, 1983.

35. Ten Kate TK: Design and implementation of an interactive karyotyping program in $\mathrm{C}$ on a Vicom digital image processor. Master's thesis, Department of Applied Physics, Delft University of Technology (Thesis supervisors: F.C.A. Groen and I.T. Young), 1985.

36. Tjio JH, Levan $\mathrm{H}$ : The chromosome number in man. Hereditas 42:1-6, 1956.

37. Mayall BH, Tucker JD, Christensen ML, van Vliet LJ, Young IT: Experience with Athena, a semi-automated karyotyping system. Cytometry, this issue.

38. Visser RT: Classification of banded chromosomes using a priori cytologic knowledge. Master's thesis, Department of Applied Physics, Delft University of Technology, (in Dutch), Master's thesis, Department of Applied Physies, Delft University of Technology (Thesis supervisor: F.C.A. Groen), 1981.

39. van Vliet LJ, Verwer BJH: A contour processing method for fast binary neighborhood operations. Pattern Recognition Letters 7 : $27-36,1988$

40. Vossepoel AM, Smeulders AWM, Van de Broek K: DIODA: Delineation and feature extraction of microscopical objects. Comput Programs Biomed 10:231-244, 1979.

41. Young IT, Groen FCA, Dorst L, Geerlings AC, Jovin T, ArndtJovin DJ: Comparative imaging and quantification of DNA species in chromosomes. Proceedings of the Vth European Chromosome Analysis Workshop, Heidelberg, West Germany, 1983.

42. Young IT, Roos R: Acuity: Image analysis for the personal computer. In: Pattern Recognition in Practice, Vol. III, Gelsema ES, Kanal LN (eds). North Holland Press, Amsterdam, 1988, pp.516.

43. Zack GW, Spriet JA, Latt SA, Granlund GH, Young IT: Automatic detection and localization of sister chromatid exchanges. J Histochem Cytochem 24(1):168-177, 1976

44. van Zee GA: Automated chromosome analysis of Feulgen stained specimens. Master's thesis, Department of Applied Physics, Delft University of Technology (in Dutch), Master's thesis, Department of Applied Physics, Delft University of Technology (Thesis supervisor: F.C.A. Groen), 1974. 